\title{
Small Intestinal Hodgkin Lymphoma
}

National Cancer Institute

\section{Source}

National Cancer Institute. Small Intestinal Hodgkin Lymphoma. NCI Thesaurus. Code C5337.

A Hodgkin lymphoma that arises from the small intestine. 